Do Japanese chi I dren wi th hi gh- funct i oni ng aut i sm spect rum di sor der $r$ espond di f f er ent I y to Wh-quest i ons and Yes/No- quest i ons

\begin{tabular}{|l|l|}
\hline 著者 & O Nanabu \\
\hline $\begin{array}{l}\text { j ournal or } \\
\text { publ i cat i on t i t l e }\end{array}$ & Cl i ni cal Li ngui st i cs and Phonet i cs \\
\hline vol une & 24 \\
\hline number & 9 \\
\hline page r ange & $691-705$ \\
\hline year & 2010-09-01 \\
\hline URL & ht t p: //hdl . handl e. net $/ 2297 / 25214$ \\
\hline
\end{tabular}




\title{
Do Japanese children with high-functioning autism spectrum disorder respond differently to Wh-questions and Yes/No-questions?
}

\author{
MANABU OI \\ United Graduate School of Child Development, Osaka University, Kanazawa University, \\ and Hamamatsu University School of Medicine, Fapan
}

(Received 6 December 2009; Accepted 21 April 2010)

\begin{abstract}
The present study compared 12 Japanese children with high-functioning autism spectrum disorder (HFASD), ranging in age from 7.3-14.8 years, with 12 typically developing (TD) children matched for age, gender, and vocabulary. The means of full-scale IQ and verbal-IQ of the children with HFASD were $95.92(\mathrm{SD}=15.30)$ and $98.00(\mathrm{SD}=18.44)$, respectively. Children responded to questions from their mothers in conversations collected under a semi-structured setting, and the responses of both groups were examined from the viewpoint of adequacy. Compared to TD children, HFASD children produced more inadequate responses to Wh-questions than to Yes/No questions. To both types of questions, HFASD children produced more inappropriate responses than TD children. The findings suggest that parents of HFASD children should consider the influence of the question format on these children's response inadequacies.
\end{abstract}

Keywords: high-functioning autism spectrum disorder, Wh-questions, Yes/No questions, fapanese

\section{Introduction}

Wh-questions (Wh-Qs) are one of the basic language structures that children need to acquire. Little attention has been paid by researchers, however, to the acquisition of Wh-Qs in children (Fletcher and MacWhinney, 2004). Little is known on this subject other than the chronological order of acquisition in early childhood: what, where, why, how, and when (Tyack and Ingram, 1977).

From the viewpoint of pragmatic development, in which children with autism have severe difficulties, responding to maternal Wh-Qs is regarded as an important opportunity for young English-speaking children to learn how to talk about past events or stories they have experienced or heard (Peterson and McCabe, 1992; 1994). Narrative language in children, adolescents, and even adults with high-functioning autism spectrum disorder (HFASD) is poorer and more problematic than in matched controls (Losh and Capps, 2003; Loveland and

Correspondence: Manabu Oi, United Graduate School of Child Development, Osaka University, Kanazawa University, and Hamamatsu University School of Medicine, B-b43, 13-1, Takaramachi, Kanazawa, 920-8640, Japan. Tel: +81-76-264-5512. Fax: +81-76-264-5510. E-mail: oimanabu@ed.kanazawa-u.ac.jp

ISSN 0269-9206 print/ISSN 1464-5076 online (C) 2010 Informa UK Ltd.

DOI: $10.3109 / 02699206.2010 .488313$ 
Tunali, 1993). Two questions arise here: how do children with HFASD respond to maternal Wh-Qs, and how do their responses to these questions relate to their narrative language? Clinicians have suspected that children with HFASD have more difficulty responding adequately to Wh-Qs than to yes/no questions (Y/N-Q). Interestingly, however, relatively few studies have been conducted on the former issue and none on the latter. Curcio and Paccia (1987) showed that children with autism who had mild-to-moderate retardation (average age 9 years, 8 months) demonstrated far more difficulties responding adequately to Wh-Qs than to Y/N-Qs. Oi (2008a) conducted conversational analysis of a 6-year-old Japanese boy with HFASD and came to the same conclusion.

Although relative difficulty of responding to Wh-Qs compared with $\mathrm{Y} / \mathrm{N}-\mathrm{Q}$ s has been suggested for children with autism, no relevant research has been conducted for children with Asperger syndrome. However, children with Asperger syndrome may experience the same difficulties for the following reasons. By school age, children with high-functioning autism and those with Asperger syndrome are not thought to vary greatly in their pragmatic ability (Ramberg, Ehlers, Johansson, and Gillberg, 1996). In addition, Howlin (2003) indicated that the differences in language ability between the two conditions decrease at $\sim 4-5$ years old. This raises the question of whether Wh-Qs are harder to respond to than $\mathrm{Y} / \mathrm{N}-\mathrm{Qs}$ for children with HFASD across diagnoses.

An essential but incredibly difficult question remaining unanswered is why these children should have more difficulty responding to Wh-Qs. Despite a few attempts (Hewitt, 1998; Louksa et al., 2007), we have made little progress in this area since an assumption made by Curcio and Paccia (1987) regarding their study in which participants were not highfunctioning, as mentioned above. They assumed that an increased need for externally imposed structure (Clark and Rutter, 1981) results in greater difficulty with Wh-Qs because the child must generate the requested information himself rather than simply affirming or negating the information provided by the adult. This explanation matches the definition of questions proposed by Searle (1969). He defined questions as attempts by a speaker to solicit information from a hearer. In $\mathrm{Y} / \mathrm{N}-\mathrm{Q}$, since the speaker does not know whether the proposition is true, the hearer is expected to give judgement on it. In Wh-Qs, since the speaker lacks information to make the proposition true, s/he inserts such a propositional function as 'what', and the hearer is expected to provide such information. However, the definition above does not include questions that require the hearer's acknowledgement, even in English, and falls short of explaining the diversity and pragmatic non-discreteness of questions in Japanese (Tsuchihashi, 1983). Furthermore, the concept of structure, although thought to underlie autism itself, remains unrefined (Clark and Rutter, 1981). Another pragmatic perspective to look at the problem has been provided by Loukusa et al. (2007). They have shown, from the viewpoint of relevance theory, that pragmatic errors produced by children with Asperger syndrome or high-functioning autism in responding to questions can be explained by utilization of irrelevant contextual factors, failure to address the focus of the question, or inability to stop processing after deriving a correct answer.

We need a syntactic as well as a pragmatic perspective to understand probable response difficulties to Wh-Qs in children with HFASD. In this regard a study conducted on children without autism can serve as a reference. Deevy and Leonard (2004) compared children with specific language impairment (SLI) and typically developing (TD) children matched on receptive vocabulary test scores on a comprehension task consisting of short and long subject and object Wh-questions. The two groups performed similarly on short questions, showing high accuracy in both subject and object conditions. However, the children with SLI showed poorer performance for long object questions compared with long subject questions. They 
were also less accurate on long object questions than TD children. Deevy and Leonard argued that the demands on linguistic processing abilities play an important role in the difficulties experienced by children with SLI. Howlin (2003) showed that scores on language comprehension and expression tests were well below chronological age level in adults with high-functioning autism and those with Asperger syndrome. Accordingly, response failure to Wh-Qs seen in children with HFASD might reflect linguistic problems. If it were confirmed that children with HFASD find it harder to answer Wh-Qs than Y/N-Qs, the next step would include examination of the relationship between syntactic and pragmatic components in contributing to this pattern. However, this is likely to be difficult because the difference in response difficulty between Wh-Qs and Y/N-Qs in children with HFASD is thought to be influenced by interaction of pragmatic and syntactic components in the question. Oi (2008a) conducted conversation analysis for a boy with HFA, focusing on responses to both types of question. The boy failed to respond adequately when asked Y/N-Qs about what his intention was, but responded successfully to $\mathrm{Y} / \mathrm{N}-\mathrm{Q}$ s which asked him whether he would perform some action. In addition, Paccia and Curcio (1982) found that echolalic children with autism (albeit not high-functioning) produce immediate echolalia more frequently to $\mathrm{Y} / \mathrm{N}-\mathrm{Q}$ s than to Wh-Qs. They attributed the relative difficulty of responding to $\mathrm{Y} / \mathrm{N}-\mathrm{Q}$ s to the fact that these questions require children to determine whether the question's predicate is true; accordingly, an appropriate response entails processing utterance-internal relations. This could occur in children with HFASD as clinicians have reported cases where the child ignores $\mathrm{Y} / \mathrm{N}-\mathrm{Q}$ s on rare occasions even when they are syntactically very simple. Hence we are no closer to answering the essential question of why children with HFASD might find Wh-Q's more difficult to answer than $\mathrm{Y} / \mathrm{N}-\mathrm{Q}$.

To investigate whether children with HFASD produce more inadequate responses to Wh-Qs than to Y/N-Qs, we need a method to evaluate the relationship between the adult's question and the child's response from the semantic and pragmatic points of view. The only such method in the literature is one devised by Bishop, Chan, Adams, Hartley, and Weir (2000), which seems very suitable for the present study. They made a distinction between adequate and inadequate responses regarding whether the required information was provided. Responses regarded as inadequate were further sub-divided into two categories: semantically inadequate and pragmatically inappropriate. We need a slight modification of their method, however. When evaluating response adequacy, Bishop et al. (2000) excluded Y/N-Qs to solicit a child's acknowledgement. However, Oi (2008a) found that the Y/N-Qs to which the boy responded adequately were all acknowledgement-soliciting ones. Hence we should evaluate the adequacy of responses to Y/NQs without distinguishing between information-soliciting and acknowledgement-soliciting types. We also need to look at probable differences between Japanese and English in terms of grammatical form of questions. We could not find clear definitions of Wh-Qs and Y/N-Qs in English; however, studies such as those of Bishop et al. and Peterson, Dowden, and Tobin (1999) have focused on the influences of adult question types on the child's response quality. As for WhQs, Japanese and English appear similar in terms of having Wh-words, although Japanese has far more of this type of questions than English. On the one hand, the two languages differ considerably when it comes to Y/N-Qs. English Y/N-Qs are simply ones starting with 'Do you', 'Can you', 'Is it', and the like, while tag questions or declaratives with questioning intonation also serve as $\mathrm{Y} /$ N-Qs. Japanese $\mathrm{Y} / \mathrm{N}-\mathrm{Qs}$ are more diverse than their English counterparts: there are about 10 sentence-final particles that make a Japanese sentence interrogative. Moreover, functions of Japanese Y/N-Qs are ambiguous and not discrete (Tsuchihashi, 1983). Tsuchihashi demonstrated that Japanese sentence final particles seem to represent the lexicalization of a nondiscrete speech act continuum between what has been traditionally labelled 'declarative' and 
'interrogative'. She isolated several observable variables which reflect: (1) the speaker's confidence in his or her knowledge or information; (2) the speaker's willingness to admit challenge to his or her knowledge; and (3) the speaker's solicitation of confirmatory or corrective responses. Furthermore, the analysis showed that the variables were mutually dependent. This allows us to avoid distinguishing between information-soliciting and acknowledgementsoliciting $\mathrm{Y} / \mathrm{N}-\mathrm{Q}$ s when evaluating response adequacy.

The next step to take will be to answer 'how do responses by children with HFASD to maternal Wh-Qs relate to their narrative language?' A post-hoc analysis of part of the present study (described in the Discussion) suggests a possible relationship between the child's responses to maternal Wh-Qs and his or her narrative language.

The present study addressed the following question: Do Japanese children with HFASD respond differently to an adult's Wh- and Y/N-Qs when compared with typically developing (TD) children?

\section{Methods}

\section{Participants}

In this study, 12 children with HFASD and 12 TD comparison children participated. The TD children were recruited through the personal networks of mothers of the HFASD children. This way of recruiting probably secured homogeneity of both groups of children in terms of their socio-economic status. Their mothers also participated as conversation partners for their own children when collecting conversation samples. All participants were native Japanese speakers from middle-class families residing in Japan. The HFASD group comprised 10 boys and two girls, ranging in age from 7.3-14.8 years (mean, 10.5; SD, 2.5), five of whom were diagnosed by a psychiatrist or a paediatrician as having Asperger syndrome and seven as having highfunctioning autism. All fulfilled the diagnostic criteria for one of the pervasive developmental disorders defined in DSM-IV-TR. Six months before the data were collected, children in the HFASD group were assessed using the third edition of the Wechsler Intelligence Scale: fullscale IQ ranged from 79-129 (mean, 95.92; SD, 15.30), and verbal-IQ from 80-136 (mean, 98.00; SD, 18.44). The TD participants, who ranged in age from 7.0-15.0 years (mean, 10.8; $\mathrm{SD}, 2.5)$, were selected individually to match those with HFASD in terms of gender, age, and vocabulary. For both groups of children, the Picture Vocabulary Test (PVT) was administered 6 months before data were collected. As six of 12 children with HFASD and eight of 12 TD children were older than 10 years, which is the upper age limit for the use of PVT, the number PVT items for which the child passed the test was calculated instead of calculating vocabulary age. The number of items passed ranged from 47-68 (maximum score on the PVT) for children with HFASD (mean, 57.2; SD, 4.11) and 48-68 for TD children (mean, 58.1; SD, 4.03). The two groups did not differ in this respect (Mann-Whitney $U=71, p=.7814$ ). The maximum PVT score was achieved by one child with HFASD and one in the TD group.

TD children's intelligence levels were not formally assessed. All of the children in both groups attended regular classes in public schools.

\section{Data collection}

Fifteen-minute conversations with each participant and his or her mother were recorded by a video camera in a quiet room of the university with a semi-structured setting designed to facilitate mother-child conversation. Each child watched an 8-minute animated cartoon 
without their mother. We then asked the mother to maintain a 5-minute conversation with the child, initiated by asking, 'Tell me, what cartoon you were watching?' We then switched to another 5-minute conversation by asking, 'What did you do yesterday?' Finally, there was free conversation for the last 5 minutes. For the first two parts of the conversation, the mothers were asked just to initiate conversation and not to pursue the topic too intensively. They were also asked beforehand to end the topic when the child initiated a new one or kept making noresponses to the mother's interrogation. A graduate student studying communication disorders transcribed the video-taped conversations. She had been trained in transcribing before the present study was conducted and was not informed of the purpose of the study. The transcribed data were supplemented by viewing the videos as needed during the later analyses.

\section{Data analyses}

The graduate student who made the transcriptions coded the data in the following three ways: (a) type of maternal question; (b) type of child's response; and (c) meshing of question and response.

Coding of maternal questions. Three types of questions were identified on the transcripts: Wh$\mathrm{Q}, \mathrm{Y} / \mathrm{N}-\mathrm{Q}$, and choice questions. The definitions were based on traditional Japanese linguistics (Kokugo-gakkai, 1972). Wh-Qs were defined as those containing at least one question word. An example of a Wh-Q in Japanese is 'Nani (what) nomu (drink)?', which could be translated into English as 'What would you like to drink?' 'Nani' is one example of a Japanese question word, of which there are more than 10.

$\mathrm{Y} / \mathrm{N}-\mathrm{Qs}$ were defined as those that could be answered within the yes/no format. This was determined by a rising contour and/or Japanese sentence-final particles such as ' $n a$ ', ' $n e$ ', ' $n o$ ', and so forth. These particles are used to request confirmation from the hearer, just as tag questions in English do. However, as mentioned above by Tsuchihashi (1983), these particles do not serve exactly the same function as tag particles in English. An example of a Y/N-Q in Japanese with a sentence-final particle is 'Kutsu (shoes or a shoe) nugu (take off) no?' which could be translated into English as 'Do I have to take my shoes off?'

Choice questions were defined as those that consist of more than two alternatives in an utterance where each one is accompanied by a rising contour. An example of a choice- $Q$ in Japanese is 'Ehon (picture book) yomu (read)? Video (a video) miru (watch)?' which could be translated into English as 'Shall I read a picture book for you? Or do you want to watch a video?'

Coding of responses. The present study adopted a slightly modified version of the coding schema for meshing devised by Bishop et al. (2000). Their entire coding system has a twolayer structure: the first layer defines the typological classification of responses as a basis for meshing analysis in the second layer, which codes question-response meshing. We started by coding the type of child response to the maternal question and proceeded to coding the meshing. There were seven response categories: (a) ignoring, (b) no response, (c) non-verbal response, (d) prosodic response, (e) minimal verbal response, (f) extended verbal response, and (g) other. The author added 'ignoring' to separate this from the original 'no response' of Bishop et al. The original 'no response' included two cases to be coded: in the first the child took up the turn after the adult's question but did not respond to it, and in the second the child remained silent for more than a second as determined by a stopwatch or held the turn by uttering 'er' or something similar. In the present study, the first was defined as 'ignoring' and 
the second was defined as 'no response'. We considered the first to be pragmatically inappropriate and the second to be either pragmatically inappropriate or semantically inadequate. The other five categories were almost identical to those of Bishop et al. with slight modifications. The definitions of the seven categories are shown in Appendix A.

Coding of meshing of questions and responses The meshing procedures developed by Bishop et al. (2000) were adapted for coding, with the exception that $\mathrm{Y} / \mathrm{N}-\mathrm{Q}$ s were not sub-divided into acknowledgement vs information-soliciting questions. There were three meshing categories: (a) adequate, (b) inadequate, and (c) pragmatically inappropriate. The definitions and examples for coding meshing are shown in Appendix B.

\section{Reliability}

To determine inter-rater reliability, the second rater, who was a licensed speech-hearing therapist, independently coded the data from two randomly selected mother-child pairs from each of the two participant groups. She coded 401 question-response pairs in terms of question type and response type and 309 in terms of meshing. Point-by-point reliability was obtained across the conversations. The percentage inter-rater agreement was computed by dividing the number of agreements by the number of agreements plus disagreements and multiplying by 100. The percentages and Kappa coefficients (Cohen, 1960) for each coding were as follows: $99 \%$ and $\kappa=.98$ for type of question, $86 \%$ and $\kappa=.79$ for type of response, and $88 \%$ and $\kappa=.56$ for meshing. These results indicated that inter-rater agreement was excellent for types of question and response and good for meshing. Tests were two-tailed except where indicated otherwise.

\section{Results}

The total number of question-response pairs extracted from the transcripts were very similar for the two groups (mean $=100.31$, SD $=28.03$ for the HFASD children and mean $=$ 101.20, $\mathrm{SD}=28.75$ for the TD children). The mean number of choice questions produced was also similar among the two groups $(1.31(\mathrm{SD}=1.65)$ for the HFASD children and 1.67 $(\mathrm{SD}=2.46)$ for the TD children; $U=80.5, p=.9087)$. Table I shows the total number of Wh-Qs and $\mathrm{Y} / \mathrm{N}-\mathrm{Q}$ s, excluding that of choice questions because the number of these was very small. The proportions of each response type and meshing type are also shown.

The proportion of Wh-Qs was significantly higher for the HFASD children than the TD children $(U=156, z=4.215, p=.000)$ with the reciprocal pattern seen for $\mathrm{Y} / \mathrm{N}-\mathrm{Q}$ s $(U=119$, $z=2.203, p=.028)$.

The proportion of each type of response was calculated for each child by dividing the number of responses produced by the child by the number of Wh-Qs or Y/N-Qs asked of the child. The proportions of $\mathrm{Y} / \mathrm{N}-\mathrm{Q}$ s yielding ignoring $(U=111, z=2.058, p=.004)$ and no response $(U=111, z=1.768, p=.039$, one-tailed) were significantly higher for the HFASD children than for the TD children. For the TD children, the proportion of questions yielding no response was significantly higher for Wh-Qs than for $\mathrm{Y} / \mathrm{N}-\mathrm{Qs}$ (Wilcoxon's $T S=8$, $z=2.039, p=.017)$. The proportion of non-verbal responses to $\mathrm{Y} / \mathrm{N}-\mathrm{Q} s$ was significantly lower for HFASD children than for TD children $(U=134.5, z=3.067, p=.002)$. In HFASD children, Wh-Qs elicited a significantly lower proportion of extended verbal responses than $\operatorname{did} \mathrm{Y} / \mathrm{N}-\mathrm{Qs}(T S=17, z=1.957, p=.003$, one-tailed). 
Table I. Proportion of response types and meshing types to Wh-Qs and Y/N-Qs.

\begin{tabular}{|c|c|c|}
\hline & Wh-Qs & $\mathrm{Y} / \mathrm{N}-\mathrm{Qs}$ \\
\hline \multicolumn{3}{|c|}{ Total number of questions asked } \\
\hline HFASD & $28.15(9.82)$ & $70.85(24.00)$ \\
\hline $\mathrm{TD}$ & $20.58(8.25)$ & $78.92(24.42)$ \\
\hline \multicolumn{3}{|c|}{ Proportion of response types } \\
\hline \multicolumn{3}{|c|}{ Ignoring } \\
\hline HFASD & $.027(.041)$ & $.042(.066)$ \\
\hline TD & $.004(.014)$ & $.003(.007)$ \\
\hline \multicolumn{3}{|l|}{ No response } \\
\hline HFASD & $.200(.131)$ & $.166(.127)$ \\
\hline TD & $.179(.154)$ & $.080(.040)$ \\
\hline \multicolumn{3}{|c|}{ Non-verbal response } \\
\hline HFASD & $.012(.019)$ & $.027(.037)$ \\
\hline TD & $.011(.021)$ & $.158(.125)$ \\
\hline \multicolumn{3}{|l|}{ Prosodic } \\
\hline HFASD & $.016(.030)$ & $.016(.025)$ \\
\hline TD & $.021(.035)$ & $.007(.010)$ \\
\hline \multicolumn{3}{|c|}{ Minimal verbal response } \\
\hline HFASD & $.539(.148)$ & $.495(.148)$ \\
\hline $\mathrm{TD}$ & $.442(.151)$ & $.484(.071)$ \\
\hline \multicolumn{3}{|c|}{ Extended verbal response } \\
\hline HFASD & $.184(.111)$ & $.256(.149)$ \\
\hline $\mathrm{TD}$ & $.183(.132)$ & $.197(.063)$ \\
\hline \multicolumn{3}{|c|}{ Proportion of meshing types } \\
\hline \multicolumn{3}{|c|}{ Adequate response } \\
\hline HFASD & $.476(.216)$ & $.685(.143)$ \\
\hline TD & $.543(.111)$ & $.786(.102)$ \\
\hline \multicolumn{3}{|c|}{ Inadequate response } \\
\hline HFASD & $.195(.120)$ & $.057(.030)$ \\
\hline TD & $.104(.075)$ & $.039(.036)$ \\
\hline \multicolumn{3}{|c|}{ Pragmatically inappropriate response } \\
\hline HFASD & $.074(.068)$ & $.063(.068)$ \\
\hline TD & $.008(.020)$ & $.009(.013)$ \\
\hline
\end{tabular}

Numbers indicate means, with SD in parentheses.

The proportions of adequate, inadequate, and pragmatically inappropriate responses to WhQs and Y/N-Qs are also shown in Table I. These proportions were calculated for each child by dividing the number of responses the child produced by the total number of Wh-Qs or Y/N-Qs asked of the child. The proportion of adequate responses to Wh-Qs was significantly lower than that to Y/N-Qs for both groups of children (HFASD: $T S=0, z=4,445, p=.000$; TD: $T S=0$, $z=4.272, p=.000)$. The proportion of inadequate responses to Wh-Qs was significantly higher for HFASD children than for TD children $(U=113, z=1.877, p=.030$, one-tailed), although that to Wh-Qs was also significantly higher than that to $\mathrm{Y} / \mathrm{N}-\mathrm{Qs}$ for both groups of children (HFASD: $T S=3, z=2.972, p=.000$; TD: $T S=8, z=2.394, p=.001$ ). The value remaining after subtraction of the proportion of inadequate responses to $\mathrm{Y} / \mathrm{N}-\mathrm{Q}$ s from the proportion of those to Wh-Qs was significantly larger for children with HFASD than TD children $(U=144, p=.000)$. The proportion of pragmatically inappropriate responses was significantly higher both to Wh-Qs $(U=138, z=3.383, p=.001)$ and Y/N-Qs $(U=121.5$, $z=2.419, p=.016)$ for HFASD children than TD children, with no difference between the two question types being seen for either group. 


\section{Discussion}

The present study addressed the question of whether Japanese children with HFASD experience more difficulty responding to Wh-Qs than to $\mathrm{Y} / \mathrm{N}-\mathrm{Q}$ s when compared with TD children. The question arose from clinicians' impressions and a few findings in the relevant literature. The answer to the question was 'yes' in some respects and 'no' in others. The findings on response inadequacy supported an affirmative answer. The rate of inadequate response to WhQs was higher than to $\mathrm{Y} / \mathrm{N}-\mathrm{Q}$ s in the HFASD children, although this was not specific to HFASD and was also seen for TD children. Crucially, however, the rate of inadequate response to Wh-Qs in children with HFASD was higher than in TD children. Such a difference was not seen for $\mathrm{Y} / \mathrm{N}-\mathrm{Q}$. Another finding supporting an affirmative answer was that the rate of extended verbal responses, in other words those that were verbally rich and adequate, to WhQs was lower than that to Y/N-Qs only in HFASD children. Bishop et al. (2000) found no differences in these factors among children with pragmatic language impairment (PLI), those with specific language impairment, chronological-age controls, and language-age controls. Thus, HFASD children might be uniquely less competent in responding adequately to Wh-Qs.

In contrast, results on ignoring, no response, and pragmatically inappropriate response suggested a negative answer to the research question. In HFASD children, the rates of ignoring and non-response to Wh-Qs did not differ from those to $\mathrm{Y} / \mathrm{N}-\mathrm{Q}$ s. In addition, there was no difference in the rate of non-response to Wh-Qs between HFASD and TD children. Instead, to Y/N-Qs, HFASD children produced a higher rate of non-response than did TD children. These results on ignoring and not responding to $\mathrm{Y} / \mathrm{N}-\mathrm{Q}$ s might reflect the present procedure in which Y/N-Qs were not sub-divided into soliciting-acknowledgement type and solicitinginformation type. Just as the PLI children in Bishop et al.'s (2000) study were, HFASD children (who tend to have severe pragmatic impairments) might have been more likely to ignore or not respond to acknowledgement-soliciting Y/N-Qs than information-soliciting Y/NQs. This is because acknowledgement-soliciting Y/N-Qs serve a social function which provides the addressee a way of indicating his or her interest to the addresser. Social deficits in children with HFASD might interfere with this process. However, the meaning of the finding that the rate of inappropriate response to Wh-Qs did not differ from that to $\mathrm{Y} / \mathrm{N}-\mathrm{Q}$ s is unclear.

When compared with TD children, those with HFSAD tended to find it more difficult to respond to Wh-Qs. However, this was not the case for all aspects of response adequacy. A question arises here as to why this occurs. The cause of the poor performance with Wh-Qs in HFASD children might be poor linguistic ability on the basis of the definitions of inadequate responses by Bishop et al. (2000), as they regarded these as vague, under-specified, or apparently reflecting poor understanding of the words in a question. However, this seems unlikely, because the number of PVT test items passed by the two groups of children did not differ, and because mean verbal-IQ was 98 for the children with HFASD and this was assumed to be similar to that for TD children. Rather, the cause could be a cognitive deficit according to the assumption by Curcio and Paccia (1987), who argued that $\mathrm{Y} / \mathrm{N}-\mathrm{Q}$ s might impose an external structure for responses, but that Wh-Qs might not impose such a structure, which children with autism have a greater need for than do TD children (Clark and Rutter, 1981). As for actually responding to Y/N-Qs, the present results showed no difference between children with HFASD and TD children in terms of the extended and minimal verbal responses which can be considered to reflect linguistic ability. This assumption can be verified by examining whether responses of children of both groups to $\mathrm{Y} / \mathrm{N}-\mathrm{Q}$ s contained grammatically complex and semantically rich language rather than being confined to 'yes' or 'no'. 
Another question arises here as to why the present participants did not show as much difficulty in responding to Wh-Qs compared with Y/N-Qs as did the subjects of Curcio and Paccia (1987). The subjects of their study failed to respond to almost all Wh-Qs from adults. The difficulties shown by the present children with HFASD are seemingly mitigated compared to those seen in subjects with mental retardation as well as autism. The present participants have probably attained higher linguistic and cognitive abilities than those studied by Curcio and Paccia, although the average age of both groups of participants was very similar. It could be hypothesized that it is easier for children with HFASD to respond to Wh-Qs than it is for children with mental retardation as well as autism, if such higher abilities decrease the need for an externally-imposed structure. This does not necessary mean, however, that children with HFASD have no need for an externally-imposed structure in responding to questions from adults. This is suggested by a preliminary study by Tanaka and Oi (2007). They showed that children with HFASD, who were very similar to those in the present study, could respond adequately to virtually all of $\sim 20 \mathrm{Wh}-\mathrm{Qs}$ presented in a cartoon. These Wh-Qs, which required contextual information contained in the plots of the cartoon, were prepared before the cartoon was watched and were presented consecutively to the children in a written questionnaire after the children had talked with their mothers about the cartoon. While Wh-Qs produced by the mothers seemed very similar to those asked in the questionnaire, children with HFASD failed the former and successfully responded to the latter. It seems likely here that the consecutiveness of Wh-Qs asked in the questionnaire following the plots of the cartoon provided an external structure which helps the child respond to questions, whereas incidental Wh-Qs asked by the mother did not. Further investigation is needed to clarify whether a question that solicited an adequate response from the child when asked in the questionnaire did not when asked incidentally in mother-child conversation.

The third question to be answered is why every Wh-Q was not difficult to respond to and why every Y/N-Q was not easy to respond to. Further investigation of this issue needs to take into account multiple factors which might contribute to determining the difficulty of questions. Hewitt (1998) showed that young adults with autism who were functioning in the mild-to-borderline range of mental retardation tended to fail to respond to Wh-Qs which were longer than seven words, had multi-clauses, had inferential requirements, or requested information indirectly. In investigating responses to $\mathrm{Y} / \mathrm{N}-\mathrm{Q}$, such a multivariate analysis must consider whether the object solicited is information or acknowledgement. Comparing contextual demands in Wh-Qs and Y/N-Qs as Loukusa et al. (2007) did might also be helpful to understand why this happened. Although the study did not involve autism, Snow (1996) sought to explain inappropriate responses to questions in children with semantic-pragmatic disorder and found a receptive linguistic explanation: comprehension failure to Wh-Q that was associated with a set of relational concepts. This suggests children with HFASD also might fail to respond to Wh-Qs with specific Wh-words. Hence differences in response adequacy among Wh-words might be found in these children. Oi (2008a) suggested another possible cause of response difficulty to Wh-Qs in children with HFASD. In his conversational analysis of a boy with HFA, he found that Wh-Qs were difficult for the boy to respond to because they required him to clarify his interpretation of an attributed thought or a desirable thought' (Happé, 1991 pp. 234-236) while Y/N-Qs were easy to respond to because they asked him to clarify 'a description of an actual state of affairs or a desirable state of affairs' (Happé, 1991, pp. 234-236). Further investigation into response difficulty to Wh-Qs should adopt a multivariate analysis approach, taking the above-mentioned variables into account.

The procedure for collecting question-response pairs should be re-evaluated in further investigations. The semi-structured settings adopted in the present study had advantages 
which were proven by the unexpected comparability of the total number of maternal questions between children with HFASD and TD children. However, the settings may have had some disadvantages. Two-thirds of the conversations collected were initiated by maternal interrogation, and such interrogation might have kept the conversations going despite the instructions given to mothers not to press the discussions. Undue focus by the mother on eliciting information from the child about the cartoon he or she watched or what happened yesterday could have reduced communicative initiation by the child. The less the child initiates, the less the mother might request clarification of the child's intents. Wh-Qs requiring clarification such as those seen in Oi's (2008a) study would appear less frequently under the conditions above. This might have lowered the rates of non-response, ignoring, inadequate response, and inappropriate response to Wh-Qs in the HFASD children. The unexpectedly small difference between the groups found in the present study might not generalize to other, less structured, settings. Having had mothers rather than experimenters ask questions might have complicated results and made the interpretation of them more difficult. Further investigations should compare the influence of both approaches on results. Matching between the two groups of children did not include reliable language development measures. The present study adopted the number of PVT test items passed by the child instead of formal vocabulary age due to the inapplicability of PVT to children older than 10 years. However, PVT-R, which did not exist when the present study was conducted, allows the assessment of older children than does PVT. PVT-R would give better vocabulary development matching in further investigation. The Japanese version of The Test for Reception of Grammar (TROG), expected to be published soon, should also be helpful in this respect.

The latter of the two questions posed at the beginning of this paper, 'How do the responses of children with HFASD to these questions relate to their narrative language?' can be tentatively answered by a post-hoc analysis of the present data. Oi (2008b) analysed relationships between response adequacy to questions and narrative language using data from the present study. Only in children with HFASD, the value remaining after subtraction of the proportion of inadequate responses to $\mathrm{Y} / \mathrm{N}-\mathrm{Qs}$ from the proportion of inadequate response to Wh-Qs was negatively correlated with age and the number of sentences produced by the child in relation to contextual information of an animated cartoon. This suggests that the greater the relative difficulty responding to Wh-Qs the poorer the narrative language ability. This finding encourages us to proceed to the next step of research in which the relationship between the ability to respond to questions and narrative language in children with HFASD will be examined.

The differences between Japanese and English in grammatical form of questions do not seem to have influenced the relative difficulty in responding to Wh-Qs compared with $\mathrm{Y} / \mathrm{N}-\mathrm{Q}$ s in children with HFASD. However, the finding that the rate of inappropriate response to Wh-Qs did not differ from that to $\mathrm{Y} / \mathrm{N}-\mathrm{Q}$ s in these children is worthy of examination in this regard. The need for further investigation of the relationship among grammatical form of questions, their pragmatic function, and difficulty responding to certain questions is suggested by Huang and Oi (in preparation) that asked the same research question as the present study in Taiwanese children. Maternal questions in Taiwanese were classified into four types; Wh-Qs, Y/N-Qs, A-not-A-Qs, and choice-Qs. A-not-A-Qs are seen in Mandarin and dialects of Chinese (Gasde, 2004). Typical Taiwanese A-not-A-Q is an utterance where an adjective is repeated twice, being conjoined by Chinese negation marker $b u$. An example of it is 'Gao bu gao?' which could be translated into English as 'High-not-high?' Wh-Qs were, of course, the most difficult to respond adequately to for Taiwanese children with HFASD. However, their results showed that Taiwanese Y/N-Qs were, unlike those in Japanese, difficult to respond adequately to for children with HFASD. On the other hand, choice-Qs which were rarely produced by Japanese mothers appeared very frequently 
in Taiwanese maternal questions and were easier for children with HFASD to respond to than Wh-Qs and $\mathrm{Y} / \mathrm{N}-\mathrm{Q}$ s. In addition, $\mathrm{A}-$ not-A-Qs, which are unique to Taiwanese, were also easier for these children than Wh-Qs and Y/N-Qs. A-not-A-Qs seem to resemble $\mathrm{Y} / \mathrm{N}-\mathrm{Q}$ s in English in terms of function, despite their grammatical form suggesting dichotomous choice-Qs such as 'Take-it-or-leave-it' in English (Loomis, Brown, Lucero, and Peterson, 1997). In future, we hope to examine what kind of components makes questions easy or hard to respond to adequately for children with HFASD by conducting cross-linguistic comparisons among various languages.

Finally, the clinical implication of the present study is that, in conversing with Japanese children with HFASD, Wh-Qs from mothers may be answered inadequately, but $\mathrm{Y} / \mathrm{N}-\mathrm{Qs}$ might help solicit adequate responses from children. The significance of this change in question type by the mother in conversation with the child with HFASD is to be investigated in relation to the development of the child's narrative language.

Declaration of interest: The authors has no conflicts of interest with anyone in regard to the contents of the article. This research was supported by a grant (The Grant-in-Aid for Scientific Research No.18330202) from the Japan Society for the Promotion of Science.

\section{References}

Bishop, D.V.M., Chan, J., Adams, C., Hartley, J., \& Weir, F. (2000). Conversational responsiveness in specific language impairment: Evidence of disproportionate pragmatic difficulties in a subset of children. Development and Psychopathology, 12, 177-199.

Clark, P., \& Rutter, M. (1981). Autistic children's responses to structure and interpersonal demands. Fournal of Autism and Developmental Disorders, 11, 201-217.

Cohen, J. (1960). A coefficient of agreement for nominal scales. Educational and Psychological Measurement, $20,37-46$.

Curcio, F., \& Paccia, J. (1987). Conversations with autistic children: Contingent relationships between features of adult input and children's response adequacy. Fournal of Autism and Developmental Disorders, 17, 81-93.

Deevy, P. \& Leonard, L. B. (2004). The comprehension of Wh- questions in children with specific language impairment. Fournal of Speech, Language, and Hearing Research, 47, 802-815.

Fletcher, P., \& MacWhinney, B. (2004). The handbook of Child Language. Oxford: Blackwell Publishing.

Gasde, H. (2004).Yes/No questions and A-not-A questions in Chinese revisited. Linguistics, 42, $293-326$.

Happé, F.G.E. (1991). The autobiographical writings of three Asperger syndrome adults: problems of interpretation and implicature for theory. In U. Frith, (Ed.), Autism and Asperger syndrome. Cambridge: Cambridge University Press.

Hewitt, L.E. (1998). Influence of question type on response adequacy in young adults with autism. Fournal of Communication Disorders, 31, 135-152.

Howlin, P. (2003). Outcome in high-functioning adults with autism with and without early language delays: implications for the differentiation between autism and Asperger syndrome. Fournal of Autism and Developmental Disorders, 33, 3-13.

Huang, S \& Oi, M. (Unpublished). Responses to maternal questions in Taiwanese children with high-functioning autism spectrum disorder: Comparison among Wh-question, Yes/No- question, Choice questions and A-not-A question.

Kokugo-Gakkai. (1972). Kokugogaku-Fiten. Tokyo: Tokyo-Dou Shuppan. In Japanese.

Loomis, J., Brown, T., Lucero, B., \& Peterson, G. (1997). Evaluating the validity of the dichotomous choice question format in contingent valuation. Environmental and resource economics, 10, 109-123.

Losh, M., \& Capps, L. (2003). Narrative ability in high-functioning children with autism or Asperger syndrome. fournal of Autism and Developmental Disorders, 33, 239-251.

Loukusa, S., Leinonen, E., Jussila, K., Matilla, M., Ryder, N., Ebeling, H., \& Moilanen, I. (2007). Answering contextually demanding questions: pragmatic errors produced by children with Asperger syndrome or highfunctioning autism. Fournal of Communication Disorders, 40, 357-381.

Loveland, K., \& Tunali, B. (1993). Narrative language in autism and theory of mind hypothesis: a wider perspective. In S. Baron-Cohen, H. Tager-Flusberg, \& D.J. Cohen (Eds.), Understanding other minds: perspectives from autism (pp 247-266). Oxford: Oxford Medical Publications. 
Oi, M. (2008a). Using question words or asking yes/no questions: failure and success in clarifying the intentions of a boy with high-functioning autism. Clinical Linguistics and Phonetics, 22, 814-823.

Oi, M. (2008b). The relationship between narrative language and responsiveness to maternal Wh-questions in Japanese children with high-functioning autism spectrum disorder. Paper presented at the 46th conference of the Fapanese Association of Special Education, Yonago.

Paccia, J., \& Curcio, F. (1982). Language processing and forms of immediate echolalia in autistic children. Fournal of Speech and Hearing Research, 25, 42-47.

Peterson, C., Dowden, C., \& Tobin, J. (1999). Interviewing preschoolers: comparisons of yes/no and Wh- questions. Law and Human Behaviour, 23, 539-555.

Peterson, C., \& McCabe, A. (1992). Parental styles of narrative elicitation: effect on children's narrative structure and content. First Language, 12, 299-321.

Peterson, C., \& McCabe, A. (1994). A social interactionist account of developing decontextualized narrative skill. Developmental Psychology, 30, 937-948.

Ramberg, C., Ehlers, A., Johansson, M., \& Gillberg, C. (1996). Language and pragmatic functions in school-age children on the autism spectrum. European Fournal of Disorders of Communication, 31, 387-414.

Searle, J. (1969). Speech acts: An essay in the philosophy of language. London: Cambridge University Press.

Snow, D. (1996). A linguistic account of a developmental, semantic-pragmatic disorder: evidence from a case study. Clinical Linguistics and Phonetics, 10, 281-298.

Tanaka, H., \& Oi, M. (2007). Retelling of an animated cartoon by children with and without high-functioning pervasive developmental disorders. Paper presented at the 32nd conference of the fapanese Association of Communication Disorders, Yokohama.

Tsuchihashi, M. (1983). The speech act continuum: an investigation of Japanese sentence final particles. Fournal of Pragmatics, 7, 361-387.

Tyack, D., \& Ingram, D. (1977). Children's production and comprehension of questions. Fournal of Child Language, 4, 211-224.

\section{Appendix A: The definitions of children's responses to questions}

The below was translated into Japanese for coding.

\section{Ignoring}

The child takes his or her turn by making a verbal response following the question, but does not answer it.

\section{No response}

The child does not respond to the question; the mother pauses for at least 1 second after asking her question, but the child either remains silent or utters a filler-like 'eh' tone (translated as 'well').

\section{Non-verbal response}

The child non-verbally responds to the question (e.g. head nodding, pointing).

\section{Prosodic response}

The child responds using such non-lexical verbal responses as 'mmh' and 'uhuh'.

\section{Minimal verbal response}

The child responds with 'yes', 'no', or 'I don't know' to a yes/no question or only provides the requested information without elaboration for a question-word question. 
Extended verbal response

The child gives a relevant response that exceeds the minimal requested information or acknowledgement.

\section{Other}

Used when no coding was applied, and the response was excluded from the meshing analysis. This was coded when the child responded to a question by challenging its presupposition, or the response was unintelligible, or an overlap between the speakers occurred.

\section{Appendix B: Guidelines for coding meshing}

Names of persons and places in the original guidelines by Bishop et al. (2000) were replaced by Japanese ones. The below was translated into Japanese for coding.

\section{Adequate response}

This was defined as one where the requested information or acknowledgement was ether provided or where the child stated that it could not be provided with a response such as 'I don't know'.

\section{Inadequate response}

This meets one of the following six conditions:

(1) a vague, over-general, or semantically under-specified response:

$\mathrm{C}$ : In the summer holidays I'm going to $\mathrm{X}$.

A: Where's that?

C: Long way away.

(2) a semantic relation error where a related word was substituted:

A: What's the doctor doing?

C: Testing the body with a 'telescope'. (referring to a stethoscope)

(3) a response to only part of an alternative question, leaving the meaning unclear:

A: And could he walk then, or did you have to carry him?

C: No.

(4) ignoring part of a complex question:

A: What is your favourite thing to drink at a party?

C: Toffees.

(5) failure of literal comprehension:

A: Where did you go on holiday?

C: In September.

(6) responding with 'I don't know' or an equivalent expression, whereas an adult would be expected to give an answer:

A: Are you going to the same place next year? 
C: Yes.

A: What's it called?

C: Can't remember.

\section{Pragmatically inappropriate response}

This meets one of the following twelve conditions:

(1) over-literal response that does not appreciate the speaker's meaning:

A: Can you tell me what sort of car your dad's got?

C: Yes. (after more than 2 seconds pause)

(2) specific referent introduced without explanation:

A: Who went with you on your holiday?

C: Taro.

A: Who's Taro?

(3) scope of question misunderstood; different type of information supplied. (These contrast with inadequate responses, where the information provided is the right kind but is too vague, general, or under-specified.)

A: So your dad drives to work every day. What sort of car has he got?

C: A brown one.

(4) hyperbolic or unbelievable response:

A: (looking at a photo of a sick child) What do you think is wrong with him?

C: He has a heart attack.

(5) failure to consider prior conversation:

A: Did you go to Kyoto in your car?

C: Yes.

A: What about when you went to Osaka?

C: It was hot.

(6) failure to use social context (These responses are often but not invariably impolite. The child's response does not take the social status of the partner into account.)

A: Who is your best friend? (the child is unfamiliar with the adult)

C: You are.

(7) unexplained self-contradiction:

A: Have you ever been to the seaside?

C: No.

A: So if you have not been to the seaside, where do you go on holiday?

C: To the seaside.

(8) apparently uncooperative use of 'I don't know'; child says 'don't know' in a context where even a young child should have no difficulty in answering the question:

A: What is your brother's name?

C: Don't know. 
(9) tangential response; the minimal response can be inferred, but only with some difficulty:
A: Have you ever been to the doctor?
C: I had an apple a day. (infer 'no')

(10) extended response that contains additional details that are irrelevant, repetitive, or bizarre:

A: What do you think is wrong with that boy?

C: I think he might have fallen into the water, January the sixth.

(11) child ignores adult and continues speaking:
A: What do you think is wrong with that boy?
C: I am having my birthday party tomorrow.

(12) inappropriately exaggerated prosody:
A: Did you have a party this year?
$\mathrm{C}$ : Oh no! (intonation suggests this is a comment on a major mishap; would be appropriate, for instance, if he had just spilled coffee on his trousers) 